Asian Spine Journal

Vol. 4, No. 2, pp 82 88, 2010

doi:10.4184/asj.2010.4.2.82

\title{
Comparison between Operated Muscular Dystrophy and Spinal Muscular Atrophy Patients in terms of Radiological, Pulmonary and Functional Outcomes
}

\author{
Hyon Su Chong, Eun Su Moon, Hak Sun Kim, Nanda Ankur, Jin Oh Park, \\ Jin Young Kim, Phillip Anthony B Kho, Seong Hwan Moon, Hwan Mo Lee, Nam Hun Seul \\ Department of Orthopaedic Surgery, Gangnam Severance Hospital, Yonsei University College of Medicine, Seoul, Korea
}

Study Design: Retrospective comparative study.

Purpose: To study and compare the surgical outcomes of muscular dystrophy (MD) and spinal muscle atrophy (SMA).

Overview of Literature: There are few reports that have evaluated and compared the surgical outcomes of MD and SMA patients.

Methods: The patients $(n=35)$ were divided into two groups: a MD group with 24 patients and a SMA group with 11 patients. The average follow-up period was 21 months. All patients were operated for scoliosis correction using posterior instrumentation and fusion. In the immediate postoperative period, all efforts were made to reduce the pulmonary complications using non-invasive positive pressure ventilation and a coughing assist devices. The patients were evaluated by radiograph in terms of the Cobb's angle, pelvic obliquity, T1 translation, thoracic kyphosis and lumbar lordosis. The pulmonary function and self-image satisfaction were also assessed.

Results: There was a lower correction rate in the MD group (41.5\%) than in the SMA group (48.3\%), even though the curves were smaller in the MD group. The correction in the pelvic obliquity was significantly better in the SMA group ( $p=0.03$ ). The predicted vital capacity showed a $4 \%$ reduction in the MD group 1 year after surgery, while the SMA group showed a $10 \%$ reduction. The peak cough flow and end tidal $\mathrm{PCO}_{2}$ did not deteriorate and were well maintained. The average score for the improvement in self-image satisfaction postoperatively was 3.96 and 4.64 for the MD and SMA groups, respectively. The total complication rate was $45.7 \% ; 14.3 \%$ of which were respiratory-related.

Conclusions: Surgical intervention for MD and SMA may be performed safely in patients with a very low forced vital capacity ( $30 \%$ ) through aggressive preoperative and postoperative rehabilitation efforts.

Key Words: Muscular dystrophy, Spinal muscular atrophy, Neuromuscular scoliosis, Surgical correction, Pulmonary function

\section{Introduction}

The progression of curves in patients with flaccid neuromuscular scoliosis, such as muscular dystrophy (MD) and spinal muscular atrophy (SMA), show a characteristically rapid progression, which can result in them being wheelchair-bound at an early age. Surgical intervention is often necessary before these curves become stiff. Early surgical correction has the definite advantage of better surgical outcomes with fewer complications [1,2]. The primary objective is to maintain the balance of the torso over a level

Received Oct 22, 2009; 1st Revised Dec 26, 2009; 2nd Revised Jan 14, 2010; Accepted Jan 14, 2010

Corresponding author: Hak Sun Kim, MD

Department of Orthopaedic Surgery, Gangnam Severance Hospital,

146-92 Dogok-dong, Gangnam-gu, Seoul 135-720, Korea

Tel: +82-2-2019-3411, Fax: +82-2-573-5393, E-mail: HAKSUNKIM@yuhs.ac 
pelvis in order to improve the functional independence and quality of life [1-9].

Many studies reported that surgical intervention improves not only the sitting balance but also the quality of life $[8$, 10-12]. However, whether surgical correction has the same beneficial effects on improving the pulmonary function is still controversial. A deterioration in the pulmonary function is a common complication in the postoperative period [4,5,13-16]. In patients with Duchenne muscular dystrophy (DMD) and SMA, the factor most closely associated with the morbidity and mortality of patients is the pulmonary function [17-19]. The probability of intraoperative and postoperative complications are increased in cases where the preoperative pulmonary function have already deteriorated, which can lead to a clinical dilemma as to whether surgical intervention should still be contemplated. Many studies focused on the pulmonary function of either patients with DMD or SMA. However, there are no studies that evaluated and compared the outcomes of these two conditions. This study examined and compared the surgical outcomes of DMD and SMA to assess the differences in the improvement of the overall sitting balance, changes in pulmonary function, and overall functional status.

\section{Materials and Methods}

Among the neuromuscular scoliosis patients treated with a surgical correction from 2002 to 2006 at our institution, only those patients with progressive flaccid neuromuscular scoliosis (MD and SMA) were enrolled in this study ( $\mathrm{n}=$ $35)$. These patients were divided into two groups: MD group $(n=24)$ and SMA group $(n=11)$. Within the MD group, there were 19 patients with DMD, 2 patients with the
Fascio-Scapular-Humeral type, 1 patient with the Becker type and 2 patients who remained unclassified. The average follow up period was 21 months (range, 12 to 54 months). Table 1 shows the demographic and surgical data for both groups.

Routine posterior instrumentation and fusion was performed using pedicle screws or sublaminar wires. Fusion up to the sacrum/pelvis was performed in 27 cases. Sacropelvic fixation using either iliac screws or Galveston was performed only for those cases with a sitting pelvic obliquity > $10^{\circ}$ using either iliac screws or a Galveston rod. In these cases, the anterior discectomy and fusion was performed prior to posterior procedures. After surgery, all patients were transferred to the intensive care unit. In the immediate postoperative period, all efforts were made to reduce the pulmonary complications using non-invasive positive pressure ventilation (NIPPV) and a coughing assist devices $[17,20]$.

\section{Radiological evaluation}

Whole spine postero-anterior and lateral radiographs were taken in either the standing or sitting position preoperatively, immediately postoperatively, and every year thereafter. The radiographic coronal plane measurement parameters included the Cobbs angle, sitting pelvic obliquity, and T1 vertebral (T1) translation. The T1 translation was measured as the horizontal offset between a vertical line drawn from the center of $\mathrm{T} 1$ to the central sacral vertical line [21]. The radiographic sagittal plane measurement parameters included the thoracic kyphosis and total lumbar lordosis measured using the Cobbs method. To avoid interobserver and intraobserver variability, all radiological measurements

Table 1. Demographic and operative data

\begin{tabular}{|c|c|c|c|c|}
\hline & Total & MD & SMA & $p$-value \\
\hline No. of cases & 35 & 24 & 11 & \\
\hline Age $(y r)$ & $12.9 \pm 3.3$ & $13.2 \pm 3.3$ & $12.3 \pm 3.2$ & 0.4397 \\
\hline Male : Female & $26: 9$ & $19: 5$ & $7: 4$ & 0.3437 \\
\hline Height $(\mathrm{cm})$ & $145.8 \pm 16.6$ & $147.5 \pm 18.3$ & $141.9 \pm 11.8$ & 0.3580 \\
\hline Weight (kg) & $37.4 \pm 11.8$ & $38.0 \pm 12.7$ & $36.2 \pm 9.7$ & 0.6711 \\
\hline Follow up (mo) & $21.2 \pm 11.0$ & $20.3 \pm 10.7$ & $23.2 \pm 11.9$ & 0.4710 \\
\hline Operation time (min) & $715 \pm 226$ & $688 \pm 228$ & $773 \pm 222$ & 0.3075 \\
\hline Blood loss (ml) & $2,488 \pm 1,281$ & $2,418 \pm 1,292$ & $2,641 \pm 1,305$ & 0.6096 \\
\hline Ventilator (day) & $2.9 \pm 3.5$ & $2.7 \pm 3.5$ & $3.4 \pm 3.7$ & 0.5951 \\
\hline ICU care (day) & $3.7 \pm 4.0$ & $3.5 \pm 3.5$ & $4.4 \pm 5.0$ & 0.5394 \\
\hline Complications (n, \%) & $16(45.7)$ & $11(45.8)$ & $5(45.5)$ & 0.9839 \\
\hline
\end{tabular}

MD: Muscular dystrophy, SMA: Spinal muscle atrophy, ICU: Intensive care unit. 
were carried out twice by two orthopedic surgeons (JYK, NHS) with the mean of four sets of values used for statistical analysis.

\section{Pulmonary function tests}

In all patients, the pulmonary function tests were carried out immediately before and after surgery, at the one year follow-up, and every year thereafter. A respiratory rehabilitation specialist at the department of rehabilitation not associated with this study assessed the respiratory function. The pulmonary function in the sitting position was evaluated using the following parameters: the vital capacity using a Micro Spirometer ${ }^{\circledR}$ (Micro medical Ltd, Rochester, UK), peak cough flow (PCF) (1/min) by ASSESS ${ }^{\circledast}$ (Health Scan Products Inc., Cedar Grove, NJ, USA), and the end tidal Pressure of $\mathrm{CO}_{2}\left(\mathrm{ET} \mathrm{PCO}_{2}\right)$ content $(\mathrm{mm} \mathrm{Hg})$ using a $\mathrm{CO}_{2}$ partial pressure meter (BCI8200 Capnocheck Capnometer ${ }^{\circledR}$, BCI International, Waukesha, WI, USA). The ability to expectorate was specifically noted and the PCF was measured because both can be useful predictors of postoperative respiratory complications. The ET $\mathrm{PCO}_{2}$ was measured to monitor for hypercapnia because it was not feasible to obtain the arterial blood samples of the patients repeatedly.

A section of the St. George's Respiratory Questionnaire (Division of Physiological Medicine, St. George's Hospital Medical School, London, UK) was used to assess the sub-

Table 2. A part of St. George's Respiratory Questionnaire

The answers below refer to activities that have typically caused you shortness of breath in the last few days

Grade 1: Sitting or lying still

Grade 2: Getting washed or dressed

Grade 3: Walking around the home

Grade 4: Walking outside on the level

Grade 5: Walking up a flight of stairs

Grade 6: Walking up hills

Grade 7: Playing sports or games jective changes in the pulmonary function (Table 2) [22,23].

\section{Functional evaluation and satisfaction}

The patients' function was measured using the Swinyard et al. [24] functional grade scale. The level of patients' satisfaction and self-image was assessed via telephone interviews using question numbers 21, 22, 23, 24 and 30 of the SRS questionnaire ver. 30 (encompassing ver. 22 and 24, modified 11/12/03).

SAS ver. 9.1 (SAS Inc., Cary, NC, USA) was used for statistical analysis. A student's $t$-test was used to compare the preoperative and postoperative data between the two groups. A $p$-value $<0.05$ considered significant.

\section{Results}

The average number of segments fused were $15.8 \pm 2.1$ (range, 11 to 19 segments) in the MD group and $16.1 \pm 1.8$ (range, 12 to 19) in the SMA group. The preoperative Cobb's angle in the MD group was $67.6 \pm 18.0^{\circ}$, which improved to $39.5 \pm 24.3^{\circ}$. On the other hand, in the SMA group, the Cobb's angle improved from $80.7 \pm 22.5^{\circ}$ to $41.7 \pm 18.7^{\circ}$ at the final follow-up. The MD group had a lower correction rate (41.5\%) than the SMA group (48.3\%) but the difference was not significant $(p=0.13$ ). The preoperative pelvic obliquity in the MD group was $19.6 \pm 10.3^{\circ}$, which improved to $10.5 \pm 5.9^{\circ}$. The SMA group showed significantly better correction from $14.3 \pm 7.9^{\circ}$ to $7.3 \pm$ $3.2^{\circ}(p=0.03)$ (Table 3). Tables 3 and 4 list the other radiological results.

Pulmonary function tests were performed on 27 and 10 patients one and two years after surgery. The vital capacity at 1 year after surgery, which was measured as a percentage of the predicted normal value according to the age and trunk height of the patient, showed a $4 \%$ and $10 \%$ decrease in the MD and SMA groups, respectively. Although the SMA group showed a greater reduction in the predicted vital

Table 3. Radiological parameters in coronal plane

\begin{tabular}{lccccccccc}
\hline \hline \multirow{2}{*}{ Cases } & \multicolumn{3}{c}{ Cobb's angle $\left({ }^{\circ}\right)$} & \multicolumn{3}{c}{ T1 tilt angle $\left({ }^{\circ}\right)$} & \multicolumn{3}{c}{ Pelvic obliquity $\left(^{\circ}\right)$} \\
\cline { 2 - 10 } & Preop & Postop & Last & Preop & Postop & Last & Preop & Postop & Last \\
\hline Total $(\mathrm{n}=35)$ & $72.0 \pm 20.2$ & $38.4 \pm 22.4$ & $40.2 \pm 22.3$ & $3.7 \pm 3.1$ & $2.3 \pm 1.7$ & $2.9 \pm 2.6$ & $17.9 \pm 9.8$ & $8.8 \pm 4.8$ & $9.4 \pm 5.4$ \\
MD $(\mathrm{n}=24)$ & $67.6 \pm 18.0$ & $38.1 \pm 24.1$ & $39.5 \pm 24.3$ & $4.4 \pm 3.6$ & $2.6 \pm 1.9$ & $3.3 \pm 3.0$ & $19.6 \pm 10.3$ & $9.3 \pm 4.9$ & $10.5 \pm 5.9$ \\
SMA (n= 11) & $80.7 \pm 22.5$ & $39.0 \pm 19.6$ & $41.7 \pm 18.7$ & $2.4 \pm 1.3$ & $1.7 \pm 1.3$ & $2.2 \pm 1.4$ & $14.3 \pm 7.9$ & $7.8 \pm 4.7$ & $7.3 \pm 3.2$ \\
$p$-value & 0.0798 & 0.9168 & 0.7942 & 0.024 & 0.183 & 0.247 & 0.1457 & 0.4117 & 0.0299 \\
\hline
\end{tabular}

Preop: Preoperative, Postop: Postoperative, Last: In last follow up, MD: Muscular dystrophy, SMA: Spinal muscle atrophy. 
capacity, the difference in the absolute vital capacity changes between the two groups was not significant ( $p>$ 0.05 ) (Table 5). On the other hand, both groups showed a similar 2-3\% decrease in the vital capacity at the end of the second year (Table 6). However, the PCF and ET $\mathrm{PCO}_{2}$ values did not deteriorate. Indeed, the PCF and ET $\mathrm{PCO}_{2}$ values measured at the end of 1 year showed little deterioration and were almost comparable to their preoperative values (Table 5). The PCF values in the SMA group at the end of 1 year even showed improvement from $179 \pm 42 \mathrm{l} / \mathrm{min}$ to $200 \pm 37$ 1/min.

Thirty-four patients, excluding one MD patient who died, were evaluated using the respiratory questionnaire both preoperatively and at the 1-year follow-up (Table 2). No significant differences were found between the two groups preoperatively and at 1 -year follow-up, and significant improve- ment in respiratory grade was observed in the entire patient population at the final follow up $(p=0.0191)$. No significant differences in the Swinyard and Deaver [24] functional grades measured for the two groups were noted preoperatively and at the 1-year follow-up. There was significant improvement in the functional grade for the entire patient population $(p=0.0314)$.

The "satisfaction with management" domain of the SRS30 patient questionnaire was assessed by questions 21, 24 and 30. The average score of this domain in the MD and SMA groups was 3.70 and 3.94, respectively, which was not statistically significant. The self-image satisfaction score was rated as 6.2/10 in the patients in the MD group and 6.9/10 in the SMA group (with an identical score of 4 for both groups). However, in response to improvement in self image postoperatively, the average score for the MD

Table 4. Radiological parameters in sagittal plane

\begin{tabular}{lcccccc}
\hline \hline \multirow{2}{*}{ Cases } & \multicolumn{3}{c}{ T-K $($ T5-T12 $)\left({ }^{\circ}\right)$} & \multicolumn{2}{c}{ L-L $\left(\right.$ L1-L5) $\left(^{\circ}\right)$} \\
\cline { 2 - 7 } & Preop & Postop & Last & Preop & Postop & Last \\
\hline Total $(\mathrm{n}=35)$ & $32.8 \pm 21.5$ & $26.2 \pm 15.8$ & $28.3 \pm 18.8$ & $13.2 \pm 23.3$ & $34.7 \pm 16.0$ & $36.2 \pm 16.0$ \\
MD $(\mathrm{n}=24)$ & $32.0 \pm 21.5$ & $23.0 \pm 15.9$ & $24.0 \pm 18.6$ & $9.4 \pm 37.3$ & $33.7 \pm 15.0$ & $34.5 \pm 14.3$ \\
SMA $(\mathrm{n}=11)$ & $34.4 \pm 22.6$ & $32.6 \pm 14.0$ & $36.8 \pm 16.8$ & $21.0 \pm 8.4$ & $36.8 \pm 18.4$ & $39.8 \pm 19.2$ \\
-value & 0.763 & 0.0986 & 0.0635 & 0.0775 & 0.6081 & 0.3744 \\
\hline
\end{tabular}

T-K: Thoracic kyphosis, L-L: Lumbar lordosis, Preop: Preoperative, Postop: Postoperative, Last: In last follow up, MD: Muscular dystrophy, SMA: Spinal muscle atrophy.

Table 5. Pulmonary function tests

\begin{tabular}{|c|c|c|c|c|c|c|c|c|}
\hline \multirow{3}{*}{ Cases } & \multicolumn{4}{|c|}{ Vital capacity } & \multicolumn{2}{|c|}{$\mathrm{PCF}$} & \multicolumn{2}{|c|}{ ET $\mathrm{PCO}_{2}$} \\
\hline & \multicolumn{2}{|c|}{ Preop } & \multicolumn{2}{|c|}{ POD 1 yr } & \multirow{2}{*}{$\begin{array}{l}\text { Preop } \\
1 / \mathrm{min}\end{array}$} & \multirow{2}{*}{$\frac{\text { POD } 1 \mathrm{yr}}{1 / \mathrm{min}}$} & \multirow{2}{*}{$\begin{array}{c}\text { Preop } \\
\mathrm{mm} \mathrm{Hg}\end{array}$} & \multirow{2}{*}{$\frac{\text { POD } 1 \mathrm{yr}}{\mathrm{mm} \mathrm{Hg}}$} \\
\hline & $\mathrm{ml}$ & $\%^{a)}$ & $\mathrm{ml}$ & $\%^{a)}$ & & & & \\
\hline $\operatorname{MD}(\mathrm{n}=19)$ & $1,342 \pm 592$ & $48 \pm 16$ & $1,290 \pm 616$ & $44 \pm 20$ & $251 \pm 69$ & $250 \pm 108$ & $39 \pm 7$ & $38 \pm 5$ \\
\hline SMA $(n=8)$ & $1,029 \pm 610$ & $40 \pm 23$ & $835 \pm 458$ & $30 \pm 14$ & $179 \pm 42$ & $200 \pm 37$ & $35 \pm 3$ & $33 \pm 5$ \\
\hline$p$-value & 0.2245 & 0.2965 & 0.0728 & 0.0787 & 0.0699 & 0.3847 & 0.1956 & 0.0264 \\
\hline
\end{tabular}

PCF: Peak cough flow, ET $\mathrm{PCO}_{2}$ : End tidal pressure of $\mathrm{CO}_{2}$, Preop: Preoperative, POD 1 yr: At 1 year postoperative, MD: Muscular dystrophy, SMA: Spinal muscle atrophy.

${ }^{\text {a) }}$ The percentage of the predicted value of vital capacity considering the patients' height.

Table 6. The change of vital capacity at 2-year follow-up

\begin{tabular}{|c|c|c|c|c|c|c|}
\hline \multirow{2}{*}{ Cases } & \multicolumn{2}{|c|}{ Preop } & \multicolumn{2}{|c|}{ POD 1yr } & \multicolumn{2}{|c|}{ POD 2 yr } \\
\hline & $\mathrm{ml}$ & $\%^{a)}$ & $\mathrm{ml}$ & $\%$ a) & $\mathrm{ml}$ & $\%$ a) \\
\hline $\mathrm{MD}(\mathrm{n}=5)$ & 1,070 & 42.1 & 1,104 & 38.7 & 1,024 & 36.7 \\
\hline $\operatorname{SMA}(n=5)$ & 1,280 & 48.1 & 964 & 34.1 & 904 & 31.1 \\
\hline
\end{tabular}

Preop: Preoperative vital capacity, POD 1 yr: Vital capacity at postoperative 1 year, POD 2 yr: Vital capacity at postoperative 2 years, MD: Muscular dystrophy, SMA: Spinal muscle atrophy.

a) The percentage of the predicted value of vital capacity considering the patients' height. 
group was 3.96 while that for the SMA group was significantly better at $4.64(p=0.01)$.

\section{Complications}

In the MD group, the respiratory complications included 2 cases of pneumonia and 1 case of atelectasis. The cardiovascular complications consisted of 1 case of cardiomyopathy, 1 case of pulmonary edema and 1 case of pericardial effusion. The neurological complications consisted of 1 case of seizure. The implant-related complications consisted of 1 case of iliac screw penetration into the pelvis. There were also 2 cases of wound infection. The gastrointestinal (GI) complications included 1 case of ileus, 2 cases of superior mesenteric artery syndrome, and 1 case of upper GI bleeding.

In the SMA group, the respiratory complications included 1 case of atelectasis and 1 case of pneumonia. The cardiovascular complications consisted of 1 case of pulmonary congestion. There were no neurological or implant-related complications. There was 1 case of a seroma and 1 case of a wound infection. The GI complications consisted of 1 case of abdominal distention and 1 case of upper GI bleeding.

Management of these complications involved removing the prominent hardware in the pelvic cavity and local debridement for the wound-related complications. GI complications were managed either conservatively, or by a gastrojejunostomy, which was required in 1 case.

The total complication rates for both groups are as follows: respiratory system complications (14.3\%), cardiovascular complications $(8.6 \%)$, GI symptoms, and wound infection (8.6\%). A total of 16 out of 35 patients (45.7\%) developed either surgical or medical complications. There was no difference between the two groups in terms of the complication rate (MD, 45.8\%; SMA, 45.5\%). There was no case of mortality in the immediate postoperative period. However, 1 case of DMD, who was followed up for 13 months after surgery died due to a sudden deterioration in cardiac function at 14 months after surgery.

\section{Discussion}

One of the important goals of surgical intervention for flaccid neuromuscular scoliosis is to achieve a proper balance and sitting posture. In this series, although the scoliosis curves were smaller in the MD group, the correction rate in the MD group (41.5\%) was lower than that in the SMA group (48.3\%), but the difference was not statistically significant $(p=0.13)$. Moreover, the correction in pelvic obliquity was significantly better in the SMA group.

Many authors have advocated surgical intervention in DMD cases when the forced vital capacity (FVC) remains > $35 \%$ of the normal predicted value. However some have reported that deformity correction can be performed safely using NIPPV in patients with a FVC as low as $20-30 \%$ [20,25]. Bach and Sabharwal [17] reported that pulmonary complications in patients with muscular disorders or respiratory failure can be reduced using NIPPV. In the present study, 9 patients ( 6 cases in MD group, 3 cases in SMA group) had a preoperative vital capacity $<30 \%$ of the predicted value (range, 14.9 to $29.8 \%$ ). These patients underwent an aggressive preoperative pulmonary rehabilitation program in conjunction with the respiration rehabilitation team at the department of rehabilitation medicine. Immediate postoperative care focused on reducing the period of mechanical ventilation through endotracheal intubation. In 23 out of 35 patients, the total period of ventilator use was less than 2 days. The patients were then subjected to early pulmonary rehabilitation using NIPPV and a coughing assist device. The authors suggested that an intensive respiratory rehabilitation played a key role in maintaining the relatively low incidence of respiratory complications (14.3\%) in this series despite the very low FVC preoperative values.

Another important goal of surgical intervention in cases of flaccid neuromuscular scoliosis is to reduce the rate of decline in pulmonary function and possibly increase the life expectancy of these patients. Kurz et al. [13] reported that as the scoliosis angle increases by $10^{\circ}$, the vital capacity decreases by $4 \%$ in DMD patients. Chng et al. [11] reported a decrease in the rate of FVC declined from $7.7 \%$ to $3.8 \%$ in SMA patients through a surgical correction of the spinal deformity. Robinson et al. [14] reported that the scoliosis angle of SMA patients was closely related to the pulmonary capacity, and that the PCF was improved by surgical correction. In addition, the surgically-induced lengthening of the thoracic cavity and the improved posture could stabilize the pulmonary capacity or even improve it in the long run $[14,15,26]$. In the present study, the SMA group showed a greater decrease in the FVC (\%) at the end of the first year, which is likely because the predicted vital capacity was affected by the increased truncal height. The SMA group showed a larger increase in truncal height due to the greater degree of correction of scoliosis and the predicted FVC 
showed a steeper inclination. The effect of the change in truncal height was not a concern at the end of the second year and both groups showed a similar decrease (2\% in the MD group and 3\% in the SMA group), which is consistent with other reports $[4,16]$.

Interestingly, unlike the vital capacity, the PCF and ET $\mathrm{PCO}_{2}$ did not show any deterioration. The PCF and ET $\mathrm{PCO}_{2}$ values measured at the end of 1 year showed very little deterioration and were comparable to their preoperative values. In addition, the respiratory questionnaires showed significant improvement in the respiratory grade of patients in both groups despite the decrease in vital capacity. The relatively well-maintained $\mathrm{PCF}$ and $\mathrm{ET} \mathrm{PCO}_{2}$ values, which are important determinants of symptomatology, might have induced these results. Therefore, it is believed that the aggressive preoperative and postoperative respiratory rehabilitation played a beneficial role in maintaining the PCF and ET $\mathrm{PCO}_{2}$.

High complications rates, ranging from $24-75 \%$, have been reported in neuromuscular scoliosis patients treated surgically. Mohamad et al. [18] reported that the overall complication rate was approximately $33.1 \%$ and that the complications usually occur in the respiratory system (19.4\%). In the current study, the post-surgical complications were approximately $45.7 \%$, with a relatively lower respiratory system rate (14.3\%).

In many studies on flaccid neuromuscular scoliosis, most patients and their guardians claimed that surgical intervention improved their function, appearance, sitting balance and overall quality of life [26]. Aprin et al. [27] reported that surgical intervention resulted in a high satisfaction level (86\%) in SMA patients and their guardians. In this study, both groups showed high levels of satisfaction post-operatively with no significant difference between them. The mean score for the improvement in the post-operative selfimage in the MD group was 3.96 while that in the SMA group was significantly better at 4.64. It is believed that this lower average score for self-image in the MD group might be due to the lower correction rates in the scoliosis curve. However, this requires further investigation with a longer follow-up.

One of the major limitations of this study was the disparity in the size of the two study groups, which made a precise statistical comparison between the two groups difficult. In addition, the follow-up period was short for some of the patients. However, these patients had rare, life-threatening conditions and such disparities were unavoidable. More- over, a direct comparison of two different diseases was not possible. Nevertheless, this study may have clinical significance in the terms of the assessment and comparison in the two representative conditions of flaccid neuromuscular scoliosis, namely MD and SMA.

\section{Conclusions}

Surgical intervention resulted in good outcomes in both groups in all aspects. There was no significant difference between the two groups in terms of the pulmonary function, satisfaction or functional grade. Patients with a very low FVC $(<30 \%)$ could be operated on safely. Moreover, aggressive preoperative and postoperative rehabilitation efforts can help maintain the PCF and ET $\mathrm{PCO}_{2}$ values in these patients.

\section{REFERENCES}

1. Cervellati S, Bettini N, Moscato M, Gusella A, Dema E, Maresi R. Surgical treatment of spinal deformities in Duchenne muscular dystrophy: a long term follow-up study. Eur Spine J 2004;13:441-8.

2. Shapiro F, Bresnan MJ. Orthopaedic management of childhood neuromuscular disease. Part 1: Spinal muscular atrophy. J Bone Joint Surg Am 1982;64:785-9.

3. Galasko CS, Williamson JB, Delaney CM. Lung function in Duchenne muscular dystrophy. Eur Spine J 1995;4:2637.

4. Kennedy JD, Staples AJ, Brook PD, et al. Effect of spinal surgery on lung function in Duchenne muscular dystrophy. Thorax 1995;50:1173-8.

5. Ramirez N, Richards BS, Warren PD, Williams GR. Complications after posterior spinal fusion in Duchenne' s muscular dystrophy. J Pediatr Orthop 1997;17:109-14.

6. Galasko CS, Delaney C, Morris P. Spinal stabilisation in Duchenne muscular dystrophy. J Bone Joint Surg Br 1992;74:210-4.

7. Karol LA. Scoliosis in patients with Duchenne muscular dystrophy. J Bone Joint Surg Am 2007;89 Suppl 1:155-62

8. Larsson EL, Aaro S, Normelli H, Oberg B. Weight distribution in the sitting position in patients with paralytic scoliosis: pre- and postoperative evaluation. Eur Spine J 2002;11:94-9.

9. Smith RM, Emans JB. Sitting balance in spinal deformity. Spine (Phila Pa 1976) 1992;17:1103-9.

10. Berven S, Bradford DS. Neuromuscular scoliosis: causes of 
deformity and principles for evaluation and management. Semin Neurol 2002;22:167-78.

11. Chng SY, Wong YQ, Hui JH, Wong HK, Ong HT, Goh DY. Pulmonary function and scoliosis in children with spinal muscular atrophy types II and III. J Paediatr Child Health 2003;39:673-6.

12. Mercado E, Alman B, Wright JG. Does spinal fusion influence quality of life in neuromuscular scoliosis? Spine (Phila Pa 1976) 2007;32(19 Suppl):S120-5.

13. Kurz LT, Mubarak SJ, Schultz P, Park SM, Leach J. Correlation of scoliosis and pulmonary function in Duchenne muscular dystrophy. J Pediatr Orthop 1983;3:347-53.

14. Robinson D, Galasko CS, Delaney C, Williamson JB, Barrie JL. Scoliosis and lung function in spinal muscular atrophy. Eur Spine J 1995;4:268-73.

15. Sucato DJ. Spine deformity in spinal muscular atrophy. J Bone Joint Surg Am 2007;89 Suppl 1:148-54.

16. Velasco MV, Colin AA, Zurakowski D, Darras BT, Shapiro F. Posterior spinal fusion for scoliosis in Duchenne muscular dystrophy diminishes the rate of respiratory decline. Spine (Phila Pa 1976) 2007;32:459-65.

17. Bach JR, Sabharwal S. High pulmonary risk scoliosis surgery: role of noninvasive ventilation and related techniques. J Spinal Disord Tech 2005;18:527-30.

18. Mohamad F, Parent S, Pawelek J, et al. Perioperative complications after surgical correction in neuromuscular scoliosis. J Pediatr Orthop 2007;27:392-7.

19. Murphy NA, Firth S, Jorgensen T, Young PC. Spinal surgery in children with idiopathic and neuromuscular scoliosis: what' s the difference? J Pediatr Orthop 2006;26:21620.
20. Gill I, Eagle M, Mehta JS, Gibson MJ, Bushby K, Bullock R. Correction of neuromuscular scoliosis in patients with preexisting respiratory failure. Spine (Phila Pa 1976) 2006;31:2478-83.

21. Gupta MC, Wijesekera S, Sossan A, et al. Reliability of radiographic parameters in neuromuscular scoliosis. Spine (Phila Pa 1976) 2007;32:691-5.

22. Kim HS, Moon ES, Park JO, et al. A change of pulmonary function in the patients with severe non-idiopathic scoliosis following reconstructive spine surgery. J Korean Soc Spine Surg 2006;13:106-13.

23. Jones PW, Quirk FH, Baveystock CM. The St George' s respiratory questionnaire. Respir Med 1991;85 Suppl B:2531.

24. Swinyard CA, Deaver GG, Greenspan L. Gradients of functional ability of importance in rehabilitation of patients with progressive muscular dystrophy and neuromuscular diseases. Arch Phys Med Rehabil 1957;38:574-9.

25. Marsh A, Edge G, Lehovsky J. Spinal fusion in patients with Duchenne's muscular dystrophy and a low forced vital capacity. Eur Spine J 2003;12:507-12.

26. Bridwell KH, Baldus C, Iffrig TM, Lenke LG, Blanke K. Process measures and patient/parent evaluation of surgical management of spinal deformities in patients with progressive flaccid neuromuscular scoliosis (Duchenne' s muscular dystrophy and spinal muscular atrophy). Spine (Phila Pa 1976) 1999;24:1300-9.

27. Aprin H, Bowen JR, MacEwen GD, Hall JE. Spine fusion in patients with spinal muscular atrophy. J Bone Joint Surg Am 1982;64:1179-87. 